\title{
Short stature in genetic syndromes: Selected issues
}

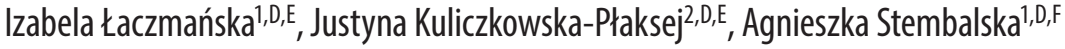 \\ ${ }^{1}$ Department of Genetics, Wroclaw Medical University, Poland \\ ${ }^{2}$ Department of Endocrinology, Diabetology and Isotope Therapy, Wroclaw Medical University, Poland \\ A - research concept and design; $B$ - collection and/or assembly of data; $C$ - data analysis and interpretation; \\ $D$ - writing the article; $E$ - critical revision of the article; $F$ - final approval of the article
}

\author{
Address for correspondence \\ Agnieszka Stembalska \\ E-mail:agnieszka.stembalska@umed.wroc.pl \\ Funding sources \\ None declared \\ Conflict of interest \\ None declared

\section{Acknowledgements} \\ The authors are grateful to Dr. Błażej Misiak for his \\ support in preparing the manuscript.
}

Received on April 25, 2016

Reviewed on June 23, 2016

Accepted on November 15, 2016

\begin{abstract}
Short stature, which is defined as height below 2 standard deviations of the mean height for the age and sex, is one of the most frequent reasons for medical consultations in children. Short stature may occur due to a constitutional delay in growth, familial short stature or chronic diseases, including many genetic syndromes, metabolic and endocrine disorders. In this article the authors provide a mini-review of the most frequent genetic syndromes associated with short stature that should be taken into account in the differential diagnosis process. Syndromes caused by chromosomal aberrations and gene mutations were divided into 2 main groups: syndromes that are associated with intrauterine growth retardation (IUGR) and those in which IUGR does not occur in the natural history of the patient. The authors described the most important anomalies in each syndrome. Metabolic diseases and skeletal dysplasias were omitted, as they are major separate groups of diseases involving growth delay.
\end{abstract}

Key words: intrauterine growth retardation, short stature, genetic syndromes

DOI

10.17219/acem/67051

\section{Copyright}

Copyright by Author(s)

This is an article distributed under the terms of the

Creative Commons Attribution Non-Commercial License

(http://creativecommons.org/licenses/by-nc-nd/4.0/) 
Short stature in children, defined as height 2 standard deviations (SD) below the mean for their age and sex, is a relatively frequent cause of referral to endocrinologists. ${ }^{1}$ Importantly, the majority of young patients have non-hormonal causes of short stature, and pediatric endocrinology should therefore be perceived as a multidisciplinary field that requires comprehensive medical knowledge and broad insight into the possible mechanisms of short stature. ${ }^{1,2}$ The causes of short stature originate from various mechanisms, many of which remain unknown. ${ }^{3}$ The most common causes include physiological variants (constitutional growth delay); familial short stature; chronic diseases with intrauterine growth restriction (IUGR), which can be accompanied by dysmorphic features; hormonal diseases; and genetic causes including chromosomal, monogenic or complex disorders. ${ }^{2}$

In this paper, we describe the most common genetic syndromes characterized by short stature that are caused by chromosomal aberrations or gene mutations. We do not go into metabolic diseases such as mucopolysaccharidoses or genetic disorders that are not syndromes, such as cystic fibrosis and skeletal dysplasias (a heterogeneous group of more than 350 disorders), which are also associated with short stature. ${ }^{4-6}$ We provide a mini-review of selected clinical issues that should improve clinical awareness of genetic syndromes associated with short stature.

\section{Genetic syndromes}

The term "genetic syndrome" refers to multiple different features that develop together and give rise to a specific clinical manifestation - a recognizable pattern of symptoms or abnormalities with one known, supposed or unknown cause. $^{3}$

According to the Winter-Baraitser Dysmorphology Database (London Medical Database), short stature has been reported in more than 1334 genetic syndromes, including 300 clinical entities with short stature characterized by prenatal onset. ${ }^{7}$

Short stature in children with syndromic diseases may be caused either by growth hormone $(\mathrm{GH})$ deficiency (hypopituitarism) or GH resistance (e.g., bone dysplasias, Laron syndrome), and also by nutritional problems with unknown mechanisms. ${ }^{8}$ In several genetic syndromes, insulin-like growth factor-1 (IGF-1) determines IUGR. ${ }^{8}$ Indeed, GH and IGF-1 are crucial for growth processes and are the final hormones in a complex network of different hormones, enzymes and receptors encoded by numerous genes in which allelic variation may contribute to the development of GH deficiency and insensitivity. ${ }^{8}$

It should be noted that when IUGR is observed during the pregnancy, the child can immediately be referred to an appropriate specialist before or after birth. Genetic disorders with short stature but without IUGR are usually diagnosed later in the patient's life. ${ }^{3}$

\section{Genetic syndromes with short stature and IUGR}

\section{Chromosomal aberrations}

\section{The 1 p36 syndrome}

The 1 p36 syndrome is the most common subtelomeric deletion syndrome, with an incidence from 1 in 5000 to one in 10,000 live births. It is characterized by intellectual disability, epilepsy, hypotonia and facial features like straight eyebrows, deep-set eyes, a flat nasal bridge, a pointed chin and midface hypoplasia. ${ }^{9}$ Growth retardation and obesity are also frequent clinical findings. Some patients present with IUGR and microcephaly. Two different phenotypes have been reported: with retarded growth and with obesity (similar to the Prader-Willi syndrome phenotype). ${ }^{10,11}$

Postnatal growth of patients with the 1 p36 syndrome does not exceed the $50^{\text {th }}$ percentile. Similarly, the mean birth weight is significantly lower than in the general population. ${ }^{9}$ Additionally, it has been reported that about $40 \%$ of patients develop various skeletal anomalies including delayed bone age, scoliosis, and asymmetry of the lower limbs. $^{12}$

\section{Monogenic diseases}

\section{The 3-M syndrome}

The 3-M syndrome is a rare autosomal recessive disorder with only a few dozen patients identified worldwide. This disorder is caused by point mutations in 1 of 3 genes: cullin 7 (CUL7, OMIM 609577), obscuring like-1 (OBSL1, OMIM $610991)$, and coiled-coil domain-containing protein-8 (CCDC8, OMIM 614145). However, mutations in other genes have also been found to act in the development of this syndrome. The 3-M syndrome is characterized by severe IUGR and postnatal growth retardation, with the mean height reaching about $120-130 \mathrm{~cm}$ in adults. Children with the 3-M syndrome have a low birth weight and delayed bone age. Characteristic features of the 3-M syndrome are a large head, short neck, prominent trapezii, a deformed sternum, short thorax, hyperlordosis, short fifth finger, prominent heels and loose joints, as well as hypogonadism in males. Notably, mental intellectual disability has not been reported in 3-M syndrome patients. ${ }^{13}$

\section{Bloom syndrome}

Bloom syndrome (BS) is an autosomal recessive disorder characterized by immunodeficiency, an increased risk of various types of cancers, and growth deficiency with severe IUGR. ${ }^{14}$ The exact incidence of BS is unknown, with about 200 cases reported; the incidence is related to ethnicity, as the syndrome is more prevalent in Ashkenazi Jews 
(one in 48,000 live births). It has been shown that mutations in the Bloom syndrome gene (BLM gene, OMIM 210900) underlie the development of BS. This gene encodes the DNA helicase that is responsible for genome stability. Mutations in the $B L M$ gene are associated with a high risk of mutations and chromosome aberrations. ${ }^{14}$ More than $90 \%$ of BS patients remain below the $5^{\text {th }}$ percentile of weight throughout their lives. More than $75 \%$ of BS patients are shorter at every age than the normal population. In addition, the patients present a low body mass index. Growth delay is the most consistent clinical finding in BS patients, while the rest of the features, such as facial teleangiectasias, immunodeficiency, and small testicular size in males, are usually not observed in infancy. ${ }^{14}$ Moreover, deceleration of skeletal growth is observed. It has been suggested that gastroaesophageal reflux, recurrent gastrointestinal infections, dietary intake, and also intrinsic immune dysfunction rather than malignancy are responsible for the patients' stunting. ${ }^{14}$

\section{Cornelia de Lange syndrome}

Cornelia de Lange syndrome (CdLS), with a frequency from 1 in 10000 to 1 in 50,000, is caused by mutations in the nipped-B-like gene (NIPBL, OMIM 608667) in 60\% of affected individuals; mutations in the structural maintenance of chromosomes 1A (SMC1A, OMIM 300040) in $5 \%$; or in the structural maintenance of chromosomes 3 (SMC3, OMIM 606062) in 1\% (genetic heterogeneity). Because of a lack of any known mutations in about $40 \%$ of patients, the role of other genes has been postulated in the etiology of this syndrome. It should be noted that a diagnosis of CdLS is based mainly on clinical manifestations. Clinical features that are characteristic of CdLS, include specific craniofacial features (microbrachycephaly, synophrys, arched eyebrows, long eyelashes, upturned nose, and widely spaced teeth), cryptorchidism, hirsutism, cardiac defects, hearing loss, myopia, and mental impairment with autistic tendencies. Growth retardation with IUGR occurs in most newborns with CdLS. Height and weight are below the $5^{\text {th }}$ percentile throughout the patient's whole life (with proportionate short stature). ${ }^{15}$

\section{Floating-Harbor syndrome}

Floating-Harbor Syndrome (FHS) is a very rare genetic syndrome (the exact frequency is unknown) characterized by low birth weight (from -3 SD to 0 SD), short stature, delayed bone age (below $-2 \mathrm{SD}$, with normalization between 6 and 12 years of age), craniofacial abnormalities, skeletal anomalies (brachydactyly, clinodactyly, short thumbs, prominent joints, clavicular abnormalities), deficits in expressive language, a high-pitched voice, and mild to moderate intellectual disability with behavioral disturbances. ${ }^{16}$ In addition, gastroesophageal reflux, renal anomalies, hearing loss, hyperopia, and/or strabismus and genital anomalies have been reported. The diagnosis is based on clinical manifestations; however, a molecular analysis of the SRCAP gene should be recommended. Heterozygous truncation mutations in exon 34 have been found in 13 unrelated patients with classical features of FHS. ${ }^{16}$ Growth delay in FHS individuals is considerable after birth (even below the $3^{\text {rd }}$ percentile), and the final height may reach the $20^{\text {th }}$ percentile. In the majority of cases, the final height is between -2 and -4 SDs (about $140-155 \mathrm{~cm}$ in adults). ${ }^{16}$

\section{Non-Mendelian inheritance}

\section{Russell-Silver syndrome}

Russell-Silver syndrome (RSS), with an incidence from one in 50,000 to one in 100,000 live births, is caused by hypomethylation of the paternal imprinting center 1 (ICR1) located on chromosome 11p15.5 in 35-50\% of patients with RSS. ICR1 regulates expression of the insulin-like growth factor-2 (IGF-2) gene. Notably, IGF-2 exerts important effects on growth, especially during fetal development. Patients with the hypomethylation defect tend to have inappropriately increased levels of IGF-1 and IGFBP-3, which might suggest a reduced sensitivity to IGF-1. ${ }^{17}$ About $10 \%$ of RSS cases are caused by a maternal uniparental disomy of chromosome 7. Other causes of RSS, such as chromosome 7 anomalies or $11 \mathrm{p} 15.5$ duplications, are rarely reported, but $40 \%$ of RSS patients have unknown disease etiology. Clinical features characteristic of RSS are IUGR $\left(<10^{\text {th }}\right.$ percentile), postnatal growth delay with height below the $3^{\text {rd }}$ percentile, facial asymmetry, a high forehead, small jaw, triangular face, clinodactyly, short arms, skeletal asymmetry, feeding disorders, and developmental delay. ${ }^{3}$ Patients with RSS have a low birth weight (about 1900-2000 g) and decreased postnatal growth, usually below the $3^{\text {rd }}$ percentile, with delayed bone age. The final height is $151.2 \mathrm{~cm}$ for men and $139.9 \mathrm{~cm}$ for women. Importantly, GH therapy might have beneficial effects in terms of improving the final height of RSS children. ${ }^{3}$

\section{Genetic syndromes with short stature without IUGR}

\section{Chromosomal aberrations}

\section{Down syndrome}

Down syndrome (DS) is the most common cause of intellectual disability regardless of gender. It is a wellcharacterized syndrome with a mean prevalence of 1 in 800 live births. The cause of DS is a trisomy of all or part of chromosome 21 (OMIM 190685). It is associated with distinctive dysmorphic features, mental retardation, growth hormone deficiency, and muscular hypotonia in infancy. In some individuals with DS, congenital malformations occur, including heart defects (in 30-40\% of DS 
patients), duodenal stenosis or atresia, imperforate anus, and Hirschsprung disease. Celiac disease, hypothyroidism, hearing and vision problems, as well as an increased risk of leukemia development are also observed. Intellectual disability, speech delay and behavioral problems are present with varying expression. ${ }^{18}$ The mean birth length in DS newborns is $48 \mathrm{~cm}$. From the age of 6 months to 3 years, the growth rate is lower than in healthy children. The final height $(161.5 \mathrm{~cm}$ and $147.5 \mathrm{~cm}$ for men and women, respectively) is reached at 15-16 years of age, with a decreased pubertal growth rate. ${ }^{19}$

\section{Prader-Willi syndrome}

Prader-Willi syndrome (PWS) is a neurodevelopmental disorder with a prevalence from 1 in 10,000 to 1 in 30,000 live births. Clinical features characteristic of PWS are muscular hypotonia, suction difficulties in infancy, then progressive obesity, short stature, hypogonadism, behavioral abnormalities, mental impairment, and sleep disturbances. Dysmorphic features observed in PWS patients include characteristic facial features (narrow bifrontal diameter, almond-shaped palpebral fissures, a narrow nasal bridge, a thin upper lip with a downturned mouth), small hands and feet and scoliosis. ${ }^{3}$ The diagnosis of PWS is based on a molecular analysis of the patient's DNA and microdeletion detection in the patient's chromosomes. The absence of paternal copies of genes localized in region 15q11-13 may be caused by microdeletion (in about $70 \%$ of PWS cases), a maternal uniparental disomy of 15q11-13 (20-25\%), or methylation silencing $(2-5 \%) .^{3}$ It is now being increasingly recognized that patients with a maternal uniparental disomy might be at risk of specific psychiatric and behavioral comorbidities, including psychotic and affective disorders, as well as autism-spectrum disorders. ${ }^{20}$

The stature of PWS patients in the first 2 years of life is below the $3^{\text {rd }}$ percentile; in the next $10-12$ years the height reaches the $10^{\text {th }}$ percentile; and after $12-14$ years of age it falls below the $5^{\text {th }}$ percentile. The average adult height is $155 \mathrm{~cm}$ and $148 \mathrm{~cm}$ for males and females, respectively. ${ }^{3}$ The cause of short stature is GH deficiency (in $80 \%$ of children with PWS, and $50 \%$ of adults), and no pubertal growth spurt. Patients with PWS have abnormal hypothalamic function with decreased secretion of hormones that regulate GH and gonadotropin production. Postmortem studies of patients with PWS have revealed decreased volumes of paraventricular nuclei and a number of oxytocin neurons. In addition, structural alterations in the region responsible for the secretion of growth hormone releasing-hormone $(\mathrm{GHRH})$ that might lead to decreased production of GH have been observed. ${ }^{3}$

\section{Turner syndrome}

Turner syndrome (TS) is caused by a lack of all or part of one X chromosome, and it occurs in 1 in 2500 liveborn females. Notably, TS is associated with growth failure (stature disproportion), cardiac anomalies (very often coarctation of the aorta), renal anomalies, pubertal delay, hypergonadotropic hypogonadism with primary amenorrhoea, lymphedema, and dysmorphic features (a webbed neck, a high-arched palate, short metacarpals, scoliosis, Madelung deformity in 7\%), hearing difficulties, hypothyroidism, and glucose intolerance. ${ }^{21}$ Some patients present developmental delays, nonverbal learning disabilities and behavioral problems, but these features vary among TS patients. $^{22}$

Chromosome $\mathrm{X}$ monosomy is present in about 70\% of TS cases and is of maternal origin. ${ }^{21}$ In addition, specific structural chromosomal aberrations may underlie the development of TS, including an isochromosome $\mathrm{X}$, deletion in the short or long arm of an X chromosome or a ring $\mathrm{X}$ formation. Furthermore, mosaic forms with more than one population of cells (abnormal or normal 46, XX) or with sequences of chromosome Y might occur. In about $5 \%$ of TS patients the $S R Y$ gene is present, which requires additional diagnostic procedures. ${ }^{21}$

Short stature is observed in about 95\% of TS patients. The adult height, without medical intervention, reaches $143 \mathrm{~cm}$, about $20 \mathrm{~cm}$ below the normal range. It is diagnosed mainly at the age of 5 years..$^{21,22}$

\section{The $18 \mathrm{q}$-syndrome}

The 18q- syndrome is a deletion syndrome with a frequency of 1 in 40,000 live births. The structural abnormalities of 18q-include proximal interstitial deletions, complex cryptic rearrangements, and distal deletions. Most cases are sporadic, although familial cases have been also reported. It has been shown that the phenotype expression is characterized by a high inter-individual variability depending on the size of the deletion. The most typical features include growth delay accompanied by $\mathrm{GH}$ deficiency, intellectual disability, autoimmune disorders, microcephaly, midface hypoplasia, a flat philtrum, a broad nasal bridge, hearing impairment, eye anomalies, neurologic and genitourinary abnormalities. One study mapped a $2 \mathrm{Mb}$ critical region for $\mathrm{GH}$ insufficiency to the $18 \mathrm{q} 23$ locus. ${ }^{23}$ Interestingly, several genes playing an important role in $\mathrm{GH}$ response are located within this region, including the myelin basic protein gene (MBP), and the galanin receptor 1 gene (GALR1). Chromosome analysis is recommended in suspected cases, and also in parents for recurrent risk evaluation. ${ }^{24}$

\section{Monogenic diseases}

\section{Laron syndrome}

Laron syndrome (LS) is an autosomal recessive disorder with a prevalence of 1 in 1,000,000, characterized by short stature with normal or increased serum GH and low IGF-1 
levels. It is caused by mutations in the extracellular domain of the GH receptor (GHR) gene (OMIM 600946), which result in low $\mathrm{GH}$ binding protein levels and decreased IGF-1 production (OMIM 262500). The clinical features include reduced birth length without IUGR; delayed bone age and bone maturation; severe short stature; no clear pubertal spurt; occasionally blue sclerae and hip degeneration; and rarely, congenital abnormalities like strabismus, cataract or aortic stenosis occur. Sleep disorders, obesity, delayed dentition, and genital anomalies may also occur. ${ }^{25,26}$ Postnatal growth is slowed and disproportional. Adult stature ranges from -3 to -12 SD (OMIM 262500). The final mean height is $119 \mathrm{~cm}$ in females and $124 \mathrm{~cm}$ in males. ${ }^{27}$

\section{Leri-Weill syndrome}

Leri-Weill dyschondrosteosis (LWD) is a dominantly inherited disorder with a prevalence of 1 in 2000 live births. It originates from a homozygous defect in the short stature homeobox gene (SHOX, OMIM 312865) or the short stature homeobox Y-linked gene (SHOXY, OMIM 400020). Dysmorphic features in LWD include short stature, mesomelia and Madelung wrist deformation with deformity of the distal radius, ulna and proximal carpal bones (OMIM 127300). Stature is decreased for both sexes ( -2.3 SDs for girls and -1.8 SDs for boys). In addition, decreased arm spans might appear (-3.2 SDs for girls and -2.3 SDs for boys) connected with early development of mesomelia in the arms. ${ }^{28}$

\section{Noonan syndrome}

Noonan syndrome (NS) is an autosomal dominant disorder and one of the most common genetic syndromes, with a prevalence from 1 in 1000 to 1 in 2500 live births. ${ }^{29}$ About $20 \%$ of NS cases are familial. A diagnosis of NS is based mainly on clinical manifestations and genetic screening for mutations in several genes, including protein tyrosine phosphatase non-receptor type 11 (PTPN11, OMIM 176876), son of sevenless drosophila homolog 1 (SOS1, OMIM 182530), V-RAF-1 murine leukemia viral oncogene homolog 1 (RAF1, OMIM 164760), V-KI-RAS2 kirsten rat sarcoma viral oncogene homolog (KRAS, OMIM 190070), V-RAF murine sarcoma viral oncogene homolog B1 (BRAF, OMIM 164757), neuroblastoma Ras viral oncogene homo$\log (N R A S$, OMIM 164790), and mitogen-activated protein kinase 1 (MAP2K1, OMIM 176872). However, it should be noted that negative results of molecular tests do not exclude a diagnosis of NS due to considerable genetic heterogeneity. ${ }^{30}$ Children with mutations in the PTPN11 gene have mild GH resistance. Importantly, NS is characterized by distinctive dysmorphic features and congenital anomalies such as short stature, a characteristic facial appearance (hypertelorism, ptosis, low-set and posteriorly rotated ears), congenital heart defects (pulmonary valve stenosis with dysplastic leaflets in $50 \%$ of NS cases and hypertrophic obstructive cardiomyopathy in 20\%), as well as chest and spinal deformaties. Mild intellectual disability, learning difficulties, feeding problems in early childhood, hearing defects, cryptorchidism, and abnormal pigmentation are also frequently observed in NS patients. ${ }^{6}$ Final height is about $161-167 \mathrm{~cm}$ in males and $150-155 \mathrm{~cm}$ in females. More than $50 \%$ of females and $40 \%$ of males are below the $3^{\text {rd }}$ percentile, but about $30 \%$ of NS patients have normal height in adulthood. ${ }^{31}$

\section{Other syndromes}

Because of its high frequency (8 in 1000 live births), fetal alcohol syndrome (FAS) is another disorder associated with short stature that should be taken into account in differential diagnosis. The biological pathway responsible for FAS development is still unknown, but the role of epigenetic mechanisms is postulated. ${ }^{32}$

This syndrome is caused by alcohol exposure in pregnancy and has lifelong consequences for the affected child and the whole family. It is characterized by facial anomalies (smoothing of the philtrum, a thin upper lip, short palpebral fissure length), delayed bone age, short stature, functional impairments, behavioral problems, and intellectual disability. The nervous system injury caused by alcohol in the fetal period is irreversible. ${ }^{33}$

The growth of FAS children is delayed compared to the healthy population. Also, IUGR is observed and body length at birth is low. A body mass index below the $3^{\text {rd }}$ percentile is reported in $22 \%$ of FAS children, in comparison to $3 \%$ in healthy children. ${ }^{34}$ Abnormalities in the central nervous system may be manifested by microcephaly.

A diagnosis of FAS syndrome requires the involvement of a variety of professional medical specialists and often encounters considerable diagnostic difficulties. Due to the social stigma, information about alcohol consumption in pregnancy is often concealed from medical professionals. $^{33}$

\section{Conclusions}

Short stature can be a physiological feature without further implications; however, it is also one of the most frequent clinical characteristics in a number of genetic syndromes and other diseases. Assessment of growth delay should include the use of sex-specific reference data for standing height, head circumference and weight, as well as anteroposterior radiography of the left hand and wrist to determine skeletal maturation (bone age). Because it is easy to detect, primary care physicians usually refer young patients with short stature to endocrinologists and/ or geneticists to complete the diagnostic process. A great deal of attention and a detailed clinical investigation should be offered to all patients with short stature. 
Indications for therapy with recombinant GH vary depending on the country of origin. In Poland, GH therapy is reimbursed in cases of short stature associated with PWS, TS, hypopituitarism with hyposomatotropinemia and chronic renal insufficiency. In the USA, GH therapy is also indicated in cases of IUGR, NS and idiopathic short stature $<-2.25 \mathrm{SD}$. Recombinant GH therapy in skeletal dysplasias are still under investigation, but several studies from Japan argue for efficacy of this approach. ${ }^{35}$

Children with genetic syndromes, very often associated with intellectual disability and dysmorphic features, are usually first referred to clinical geneticists and after that to other specialists, among them pediatric endocrinologists. However, when clinical features other than short stature are mildly expressed, some patients with genetic syndromes are first diagnosed by endocrinologists. A diagnosis of short stature in children should be based on extensive cooperation between clinical geneticists and pediatric endocrinologists.

\section{References}

1. Johnston Rohrbasser LB. Genetic testing of the short child. Horm Res Paediatr. 2011;76(3):13-16.

2. Petriczko E, Horodnicka-Jozwa A, Walczak M. Diagnostic evaluation before growth hormone deficiency recognition (exclusion criteria). Endokrynol Pediatr. 2009;9:13-22.

3. Siklar Z, Berberoglu M. Syndromic disorders with short stature. JClin Res Pediatr Endocrinol. 2014;6(1):1-8.

4. Krakow D, Rimoin DL. The skeletal dysplasias. Genet Med. 2010;12(6): 327-341.

5. Muenzer J. Overview of the mucopolysaccharidoses. Rheumatology (Oxford). 2011;50(5):v4-12.

6. Phung OJ, Coleman $\mathrm{Cl}$, Baker EL, et al. Recombinant human growth hormone in the treatment of patients with cystic fibrosis. Pediatrics. 2010;26(5):e1211-1226.

7. Winter-Baraitser Dysmorphology Database (London Medical Database) http://www.Imdatabases.com/ Accessed April 18, 2016.

8. Kelnar CJ. Growth hormone therapy for syndromic disorders. Clin Endocrinol (Oxf). 2003;59(1):12-21.

9. Sangu N, Shimojima K, Shimada S, Ando T, Yamamoto T. Growth patterns of patients with $1 \mathrm{p} 36$ deletion syndrome. Congenit Anom (Kyoto). 2014;54(2):82-86.

10. Gajecka M, Mackay KL, Shaffer LG. Monosomy 1 p36 deletion syndrome. Am J Med Genet C Semin Med Genet. 2007;15:145C(4), 346-356.

11. Keppler-Noreuil KM, Carroll AJ, Finley WH, Rutledge SL. Chromosome $1 p$ terminal deletion: Report of new findings and confirmation of two characteristic phenotypes. J Med Genet. 1995;32(8):619-622.

12. Battaglia A, Hoyme HE, Dallapiccola B, et al. Further delineation of deletion 1 p36 syndrome in 60 patients: A recognizable phenotype and common cause of developmental delay and mental retardation. Pediatrics. 2008;121(2):404-410.

13. Holder-Espinasse M. 3-M Syndrome. In: Pagon RA, Adam MP, Ardinger HH, Bird TD, Dolan CR, Fong CT, Smith RJH, Stephens K. GeneReviews $^{\mathbb{B}}$ [Internet]. Seattle (WA): University of Washington; 1993-2014. Published March 25, 2002. Updated January 26, 2012. Accessed April 18, 2016.

14. Keller C, Keller KR, Shew SB, Plon SE. Growth deficiency and malnutrition in Bloom syndrome. J Pediatr. 1999;134(4):472-479.

15. Boyle MI, Jespersgaard C, Brøndum-Nielsen K, Bisgaard AM, Tümer Z. Cornelia de Lange syndrome. Clin Genet. 2015;88(1):1-12.

16. Nikkel SM, Dauber A, de Munnik S, et al. The phenotype of FloatingHarbor syndrome: Clinical characterization of 52 individuals with mutations in exon 34 of SRCAP. Orphanet J Rare Dis. 2013;8:63.

17. Iliev DI, Kannenberg K, Weber K, Binder G. IGF-I sensitivity in SilverRussell syndrome with IGF2/H19 hypomethylation. Growth Horm IGF Res. 2014;24(5):187-191.
18. Roizen NJ, Patterson D. Down's syndrome. Lancet. 2003;361(9365): 1281-1289.

19. Myrelid A, Gustafsson J, Ollars B, Annerén G. Growth charts for Down's syndrome from birth to 18 years of age. Arch Dis Child. 2002;87(2): 97-103.

20. Krefft M, Frydecka D, Adamowski T, Misiak B. From Prader-Willi syndrome to psychosis: Translating parent-of-origin effects into schizophrenia research. Epigenomics. 2014;6(6):677-688.

21. Gawlik A, Malecka-Tendera E. Transitions in endocrinology: Treatment of Turner's syndrome during transition. Eur J Endocrinol. 2013;170(2):R57-74.

22. Pinsker JE. Clinical review: Turner syndrome: Updating the paradigm of clinical care. J Clin Endocrinol Metab. 2012;97(6):E994-1003.

23. Cody JD, Hale DE, Brkanac Z, Kaye Cl, Leach RJ. Growth hormone insufficiency associated with haploinsufficiency at 18q23. Am J Med Genet. 1997;71(4):420-425.

24. Linnankivi T, Tienari P, Somer M, et al. 18q deletions: Clinical, molecular, and brain MRI findings of 14 individuals. Am J Med Genet A. 2006; 140(4):331-339.

25. Laron Z, Kauli R. Fifty seven years of follow-up of the Israeli cohort of Laron Syndrome patients - from discovery to treatment. Growth Horm IGF Res. 2016;28:53-56. doi: 10.1016/j.ghir.2015.08.004

26. Goswami S, Ghosh S, Chowdhury S. Growth hormone insensitivity syndrome: A sensitive approach. Indian JEndocrinol Metab. 2012;16(5): 817-818.

27. Laron Z, Lilos $P$, Klinger B. Growth curves for Laron syndrome. Arch Dis Child. 1993;68(6):768-770.

28. Ross JL, Kowal K, Quigley CA, et al. The phenotype of short stature homeobox gene (SHOX) deficiency in childhood: Contrasting children with Leri-Weill dyschondrosteosis and Turner syndrome. J Pediatr. 2005;147(4):499-507.

29. Romano AA, Allanson JE, Dahlgren J, et al. Noonan syndrome: Clinical features, diagnosis, and management guidelines. Pediatrics. 2010; 126(4):746-759

30. Agarwal P, Philip R, Gutch M, Gupta KK. The other side of Turner's: Noonan's syndrome. Indian J Endocrinol Metab. 2013;17(5):794-798.

31. Roberts AE, Allanson JE, Tartaglia M, Gelb BD. Noonan syndrome. Lancet. 2013;381(9863):333-42.

32. Varadinova M, Boyadjieva N. Epigenetic mechanisms: A possible link between autism spectrum disorders and fetal alcohol spectrum disorders. Pharmacol Res. 2015;102:71-80.

33. Landgraf MN, Nothacker M, Kopp IB, Heinen F. The diagnosis of fetal alcohol syndrome. Dtsch Arztebl Int. 2013;110(42):703-710.

34. Klug MG, Burd L, Martsolf JT, Ebertowski M. Body mass index in fetal alcohol syndrome. Neurotoxicol Teratol. 2003;25:689-696.

35. Kanazawa H, Tanaka H, Inoue M, Yamanaka Y, Namba N, Seino Y. Efficacy of growth hormone therapy for patients with skeletal dysplasia. J Bone Miner Metab. 2003;21(5):307-310. 